\title{
A Research Study on Externalization of Tacit Knowledge Based on Web2.0
}

\author{
Gang Li 1, Kun Lu 2 \\ 1Wuhan University, School of Information Management, E- \\ mail :imiswhu@yahoo.com.cn \\ 2 Wuhan University, School of Information Management, \\ E-mail: ghostk.student@gmail.com
}

\begin{abstract}
In this era of knowledge economy, knowledge management, the key point of which is externalization of tacit knowledge, has been drawing more and more attention. By using the new concepts and tools emerging after Web 2.0 this paper provides a conceptual model to externalize and utilize of tacit knowledge on the basis of analysis and cogitation of difficult key issues during the process of externalization.
\end{abstract}

\section{Introduction}

The era of internet is characterized with knowledge explosion, last half century during which people have created more knowledge than ever before has witnessed the flourish in the human civilization. More and more knowledge is being created at a fast pace. There are two kinds of knowledge which are explicit knowledge and implicit knowledge. The explicit knowledge is knowledge that has been or can be articulated, codified, and stored in certain media and can be readily transmitted to others while implicit knowledge also called tacit knowledge is knowledge that people carry in their minds and is, therefore, difficult to access. Often, people are not aware of the knowledge they possess or how it can be valuable to others, just as Michael Polanyi said "we know more than we can tell" Tacit knowledge accounting for the major part of the repository of human knowledge is often gained through the experience of individual or organization, thus, it is extremely personal knowledge which is difficult for sharing or utilization. In virtue of the new concepts and tools emerging after Web2.0 this paper provides a conceptual model for utilization of tacit knowledge based on analysis and cogitation of the difficult key issues during the process of externalization of tacit knowledge.

\section{An Analysis of Externalization of Tacit Knowledge}

In the past people have placed much emphasis on explicit knowledge but ignored the importance of tacit knowledge. Actually, tacit knowledge often turns out to be the most valuable knowledge in an organization, the research on tacit

Please use the following format when ciling this chapter:

Ii, G., Iu, K., 2007, in II:IP International lederation for Information Processing, Volume 252, Integration and Innovation Orient to F-Society Volume 2, eds. Wang, W., (Boston: Springer), pp. 60-67. 
knowledge can not only help to exploit and utilize the human's knowledge to larger extend but better extract knowledge from people, which is conducive to knowledge share. Since more and more researchers began to realize the importance of tacit knowledge, the transformation between explicit knowledge and tacit knowledge aroused increasing attention. Some researchers maintain that tacit knowledge can not be transformed into explicit knowledge, the psychologists Wagner and Sternberg are the supporters of this theory while some other researchers think the transformation between two kinds of knowledge is available, and this is typically supported by the SECI model provided by Nonaka which named the transformation from tacit knowledge to explicit knowledge externalization. This paper holds the opinion that tacit knowledge can be transformed into explicit knowledge, and the process of transformation is universal, however, the transformation seldom happens due to the characteristics of tacit knowledge. As a result, the major of tacit knowledge still exists in human's brains and is hard to share and utilize.

\subsection{Obstacles of Externalization}

In the previous part we have discussed the possibility and importance of the externalization of tacit knowledge. And in this section, this paper will analyze the obstacles which prevent the externalization:

$>$ Tacit knowledge is difficult to articulate

Tacit knowledge is difficult to articulate. It comes from the practical experience of people, and thus it is extremely individual knowledge. It is this intrinsic character of tacit knowledge that makes the externalization process so difficult.

$>$ Barrier in the subjective intention

Knowledge management encourages knowledge share which is altruism behavior. Notwithstanding, people pay prices to gain knowledge so as to take advantages over other competitors. They will certainly think that knowledge is power. Thinking of their self-interest, people are resistant to share knowledge with others. This is the barrier in the subjective intention.

$>$ Lack of platform and environment

Tacit knowledge is intangible. Sometimes it is the inspiration fleeting across our mind. And if there is no available platform to record this ephemeral knowledge resource it may well hides again. Unfortunately, many organizations are lack of this kind of platform which is convenient to grasp and record the ephemeral knowledge and able to provide an access to the share of tacit knowledge.

$>$ Lack of insurance mechanism of credit

Knowledge share requires credit without which nobody would be willing to share and communicate knowledge with others. When the concept of credit is established, people will believe each other, staff are willing to contribute their knowledge and all of them will benefit. When this share action becomes common regulations, one will maximize the use value of knowledge and build his reputation by offering his knowledge to others. However, this insurance mechanism is still imperfect which restrains staff's activity to share knowledge. 


\section{An Analysis of Web2.0 and Its Tools}

\subsection{An Analysis of Web2.0}

The concept of "web 2.0" began with a conference brainstorming session between O'Reilly and MediaLive International. Like many important concepts, Web 2.0 doesn't have a hard boundary, but rather, a gravitational core. That is to say we can not exactly define what is web 2.0 but we tell whether a web product belongs to it. We can formulate the sense of Web 2.0 by examples:

Table 1: what is Web 2.0

$\begin{array}{rll}\text { Web 1.0 } & & \text { Web 2.0 } \\ \text { DoubleClick } & --> & \text { Google AdSense } \\ \text { Ofoto } & --> & \text { Flickr } \\ \text { Akamai } & - \text { > } & \text { BitTorrent } \\ \text { mp3.com } & - \text {-> } & \text { Napster } \\ \text { Britannica Online } & - \text {-> } & \text { Wikipedia } \\ \text { personal websites } & --> & \text { Blogging } \\ \text { evite } & --> & \text { upcoming.org and EVDB } \\ \text { domain name speculation } & --> & \text { search engine optimization } \\ \text { page views } & --> & \text { cost per click } \\ \text { screen scraping } & --> & \text { web services } \\ \text { publishing } & --> & \text { Participation } \\ \text { content management } & --> & \text { Wikis } \\ \text { systems } & & \text { tagging ("folksonomy") } \\ \text { directories (taxonomy) } & -> & \text { Syndication } \\ \text { stickiness } & --> & \end{array}$

Generally speaking, Web2.0 is an outcome of the integration of new technology and concepts. It is a new application on the internet which appreciates all kinds of new understanding for internet, such as the web as platform, using the wisdom of crowds, long tail and so on. If a web service is based on these new understandings, then it is web 2.0 service. The transformation from web 1.0 to web 2.0 includes transformations from absolutely "reading" to "writing", from passively accepting to positive creating the information on internet. With respect to the fundamental element, the web page is for Web1.0 and the publishing or recording information on the internet for Web2.0; With respect to mechanism, the "Client Server" is for Web1.0 and the "Web Services" for Web2.0; In the environment of Web2.0 the web authors expand from experts to common users. Web2.0 emphasizes two core aspects, one is microcontent, the other is user. The microcontent is all the data created by users, no matter how trivial it is. For example, a web log, comments, pictures, bookmarks, favorite music lists, new friends and so on. All the microcontent is fraught with our work, everyday life and study, and it makes as much sense, if not more, as those formal documentations such as papers, books and journals do in the aspects of importance, amount and effects. On the other hand, Web2.0 gives identity to its user. In the era of Webl.0, users are not identified; it is an abstract representative of crowds. When it comes to Web2.0, web users are 
embodied and given to identifications just as our real world. These users will no longer feel they are the standers-by, which is conducive to arouse their sense of ownership and encourage them to contribute their wisdom.

\subsection{An Analysis of Web2.0 Tools}

It is determined by the intrinsic characters of Web2.0 that we can use it for the externalization of tacit knowledge in an organization. The following paragraphs will analyze three typical tools of Web2.0---Blog, RSS and Wikipedia in order to illustrate the advantages and feasibility of using Web2.0 tools to externalize and utilize tacit knowledge.

\subsubsection{Blog}

Blog is a web log system which is based on the microcontent concept. Usually we call the blog users bloggers. Bloggers can get access and create links to each other, they make comments to others blog and communicate their ideas. In virtue of co-linking, visiting others' blog or being visited by others, commenting and communicating, a certain social web is established among these bloggers which is called blogosphere. The reasons why choosing blog as tools for externalization are explained as follows:

$>$ As a typical application of Web 2.0, blog is different from personal home page in many aspects. Firstly, personal home page requires more technology knowledge and costs than a blog. A blogger can easily install a blog without any payment or technological knowledge about web such as HTML. Secondly, the primary goal of the personal home page is to provide a platform to display web users. Thus, a personal home page does more in displaying rather than communicating. However, blog is used to communicate which lays more attentions on knowledge accumulation, share and communication. And it focuses on the content rather than appearance, which makes it as a personal knowledge management system.

$>$ Blog is based on the concept of microcontent. Compared with traditional formal documentation, blog has its advantages in flexibility and convenience which is extremely beneficial for externalize staff's tacit knowledge step by step. Therefore, blog can do a better job than other traditional documentation does in exploiting tacit knowledge which is hard to express and easy to slip away from our minds. Moreover, in the era of knowledge economy, knowledge are widely scattered among various people from different industries. Some of them may be specializing in research, whose knowledge is more systematic. They are more likely to give out a complete theoretical system via formal documentations such as books, papers and periodicals. Since in most situations, knowledge is mastered by those who are not specialists, they may have some trivial ideas or scattered knowledge which is unable to be published and conveyed by formal documentation. And blog is effective for recording and collecting these knowledge fractions which may cover a plethora of knowledge.

$>$ As a web service, blog is maintained by the BSP(blog service provider), which is free for its users. We don't have to think of how it works, but just use it to satisfy our needs. Being transplanted into the Web 2.0 concept, blog service is designed to most major part of crowds. It is a fool-proof application that is easy 
to manipulate and popularize.

Blog is not only technique tool but also social channel. It may seem like a trivial piece of functionality now, but it was effectively the device that turned web logs from an ease-of-publishing phenomenon into a conversational mass of overlapping communities. For the first time it became relatively easy to gesture directly at a highly specific post on someone else's site and talk about it. Discussion emerged, chat emerged. And - as a result - friendships emerged or became more entrenched. Finally, a new social web blogosphere established and endow it with certain social attributes facilitating knowledge sparkle.

\subsubsection{RSS}

RSS was born in 1997 out of the confluence of Dave Winer's "Really Simple Syndication" technology, used to push out blog updates, and Netscape's "Rich Site Summary", which allowed users to create custom Netscape home pages with regularly updated data flows. Netscape lost its interest in this field, and the technology was carried forward by blogging pioneer Userland, Winer's company. In the current crop of applications, we see, though, the heritage of both parents. Nowadays, we use RSS to aggregate information across different sites. Via RSS someone would be able to link not just to a page, but to subscribe to it with notification every time that page changes. The following figure demonstrates how RSS works:

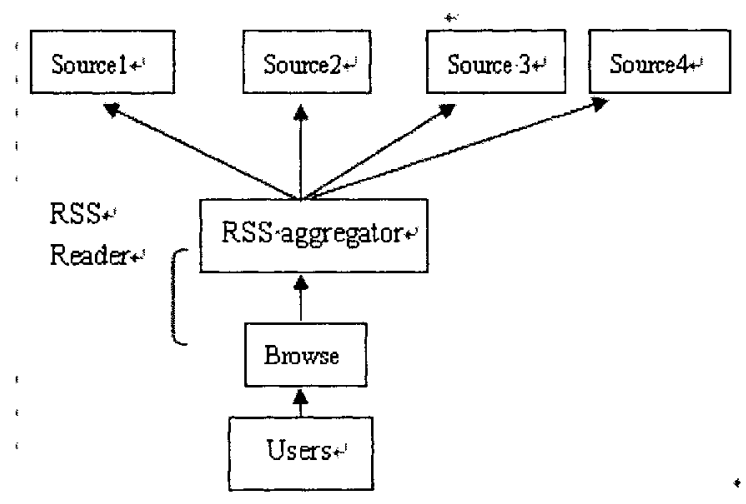

Fig.1: Mechanism of RSS

As the above figure shows, RSS can collect information from different sites and then reorganize them. RSS can also notice the update of sites and provide latest information for its users. On the other hand, users can choose and subscribe whatever they are interested in RSS reader. By virtue of RSS, web users can enjoy various information sources from different sites from only one window that is RSS reader. 


\subsubsection{Wikipedia}

Wikipedia is also a typical application of Web 2.0 ; it is a free and open collaborative encyclopedia project. In the wikipedia every entry can be added by any web user and edited by any other, which is fully based on the application of Eric Raymond's dictum that "with enough eyeballs, all bugs are shallow". It is the freedom and equality, trust and self-discipline, collaboration and share make up of the core value of wikipedia, a new type of encyclopedia facing the common crowds rather than elites. This kind of encyclopedia is efficient when apply to accumulate and deposit knowledge since it accepts any different view and encourage discussion which is conducive to self-improvement.

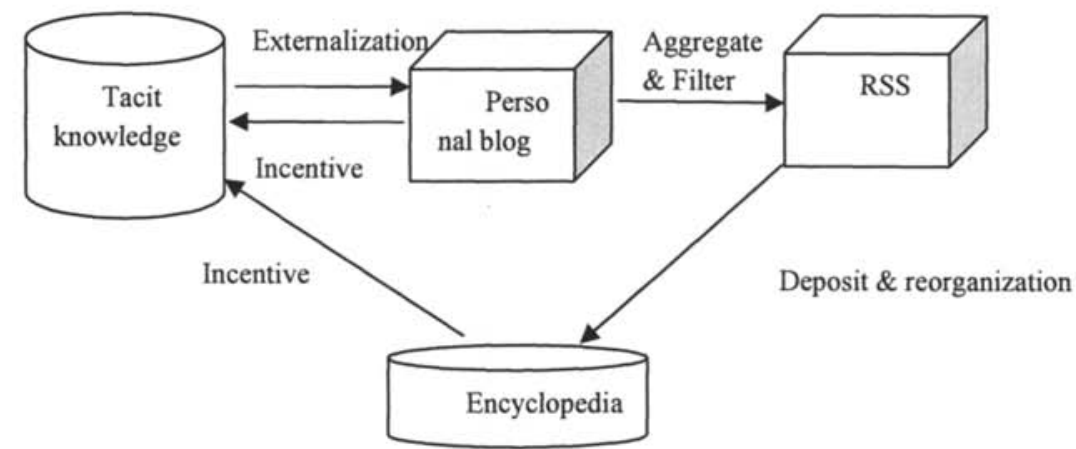

Fig.2: Conceptual model for externalization and utilization of tacit knowledge

\section{A Conceptual Model for Externalization and Utilization of Tacit Knowledge}

In the former part, we have analyzed web 2.0 and its tools. In this section we would like to give a conceptual model for externalization and utilization of tacit knowledge in virtue of the tools and concepts mentioned before. The figure below demonstrates my conceptual model of using web 2.0 for externalization and utilization of tacit knowledge:

\subsection{Externalization}

As is clearly demonstrated in the former paragraphs, blog, a typical Web2.0 application based on microcontent concept, has its natural advantages in externalizing personal tacit knowledge .In order to make full use of blog to externalize people's tacit knowledge in an organization we should first establish a personal blog system, and then encourage everyone to write blog and contribute his knowledge especially those with close relevance with his field. Make full use of blog's advantages to externalize more tacit knowledge in the organization and record it in the blog system. 


\subsection{Aggregate \& Filter}

Blog has its advantages in flexibility. However, on the other hand, knowledge externalized by blog system often appears to be so fragmentary and in low quality that we can hardly use them efficiently. Thus, we need to use RSS to aggregate all the knowledge fractions together, filter the low quality information and provide all valuable knowledge for users in a RSS reader. By aggregating and filtering, we make the knowledge externalized from blog system easier to utilize.

\subsection{Deposit \& Reorganize}

By using blog to externalization and RSS to aggregate and filter, we can get some high quality knowledge resources coming from the staff of organization which is closely relevant to survival and development of organization. And the externalized tacit knowledge is vital for an organization's core competency. Some of the knowledge may be real time knowledge which is valuable in a short time. Some others may be of long term value which can be deposited for the further use in future. Therefore, it is necessary to build an organization encyclopedia based on the concept of wikipedia.

We can extract important or long term valuable knowledge from the knowledge in RSS by further analyzing and evaluating. Then we need to reorganize this knowledge in order to make them better organized. By accumulation and deposit of this knowledge we will get a valuable knowledge repository in the organization fraught with various important, valuable and high quality knowledge. Moreover, all knowledge in the organization encyclopedia is highly relevant with the field of this organization, which generates the individual encyclopedia. And just like the wikipedia, this organization encyclopedia is also a free and open one, every staff can make comments about any entry and we can modify the entry if the comment is reasonable.

\subsection{Incentives}

To realize knowledge share within an organization, one needs not only some useful tools but to institute some regulations to catalyze this process. By establish incentive mechanism we can ensure credit of knowledge share, eliminate negative emotions preventing staff from contributing his knowledge, and enforce potential effects of organizational culture.

$>$ Quantitive incentive:

An organization should institute a serial of quantitive incentives to quantify the contribution of each staff in knowledge share. For example, how many blogs does one write in a month, how many visitors have browsed a certain blog, how many times is a blog cited by others or how many entries has one contributed to organization encyclopedia. Then corresponding reward should be provided to encourage one's contribution so as to construct a good environment for knowledge share.

$>$ Culture incentive:

The organizational culture is the view of core value in an organization. It is potential behavior regulations which restrain every person in this organization. It 
is essential to build an organizational culture which is conducive to encourage its staff to contribute and share their knowledge. Establish a common conscious that being proud with contributing knowledge and respect those who make contributions.

\section{Summary}

In this era of knowledge economy, more and more organizations began to take steps for knowledge management and utilization. If an organization wants to take precedence over others in today's intensive competition, it should handle the tacit knowledge well. This paper begins with analysis of tacit knowledge and its externalization, provides a measure for externalization and utilization of tacit knowledge in virtue of the concepts and applications of Web2.0.

\section{Acknowledgements}

The research reported in this paper is supported by the project Research on Competitive Intelligence Based on Knowledge Organization (Project No. 05JJD870159) sponsored by Key Project of Key Institution in Humane and Social Science Research, Education of Ministry of P.R. China.

\section{References}

1. Wikipedia(April 9,2007); http://en.wikipedia.org/wiki/Explicit_knowledge

2. Polanyi M. Personal Knowledge [M], Chicago: the University of Chicago Press, $1958,13-15$

3. Wagner R K, Sternberg R J. Practical in Intelligence in Real World Pursuits: The Role of Tacit Knowledge. Journal of Personality and Social Psychology, 1985(49).436458.

4. Nonaka I. A dynamic theory of organizational knowledge creation. Organization Science. 1994,5(1).14-36.

5. Tim O'Reilly. What is Web2.0 (March10,2007);

http://www.oreillynet.com/pub/a/oreilly/tim/news/2005/09/30/what-is-web-20.html 